\title{
Enhancement of Opioid-Mediated Analgesia by Ingestion of Amniotic Fluid: Onset Latency and Duration
}

\author{
JEAN C. DOERR AND MARK B. KRISTAL ${ }^{1}$ \\ Department of Psychology, SUNY at Buffalo, Buffalo, NY 14260
}

Received 21 July 1989

\begin{abstract}
DOERR, J. C. AND M. B. KRISTAL. Enhancement of opioid-mediated analgesia by ingestion of amniotic fluid: Onset latency and duration. PHYSIOL BEHAV 46(5) 913-915, 1989. - Ingestion of placenta and amniotic fluid has been shown to enhance opioid-mediated analgesia produced by morphine injection, footshock, vaginal/cervical stimulation, and during late pregnancy in rats. The present study was designed to determine how soon after ingestion the enhancement begins and how long it lasts. Tail-flick latencies in Long-Evans rats were determined before and during vaginal/cervical stimulation; analgesia was measured as the percent increase in tail-flick latency during vaginal stimulation. After determination of baseline, rats were intubated with $0.25 \mathrm{ml}$ of either amniotic fluid or beef bouillon. We found that analgesia enhancement was detectable as early as 5 minutes after ingestion of amniotic fluid, and the effect lasted at least 30 minutes, but no longer than 40 minutes.
\end{abstract}

POEF Analgesia Opioids VSIA Rats Pain Parturition Tail-flick latency Amniotic fluid

PLACENTOPHAGIA, ingestion of placenta and amniotic fluid, is observed during parturition in most placental mammals (5). Perhaps the major consequence of this behavior is the enhancement of opioid-mediated analgesia. Enhancement produced by ingestion of either amniotic fluid or placenta has been documented with analgesia produced by injection of morphine sulfate $(6,7,9)$, footshock (9), vaginal/cervical stimulation (10), and late pregnancy (8). Placenta ingestion or amniotic fluid ingestion in the absence of opioid-mediated analgesia, however, does not produce analgesia $(7,10)$. The enhancement has been shown to be dose dependent, in that a low dose of placenta or amniotic fluid enhances opioid-mediated analgesia, whereas a high dose of these substances attenuates the analgesia (6). Reversal of the enhancement effect by naltrexone $(7,9)$ suggests that the effect of placenta and amniotic fluid ingestion is specific for opioid-mediated phenomena. We have, therefore, named the active substance(s) in placenta and amniotic fluid POEF, for Placental Opioid-Enhancing Factor (6).

The present study was conducted to determine how soon after ingestion of amniotic fluid we can detect enhancement of vaginal/ cervical stimulation-induced analgesia (VSIA), and how long the enhancement lasts. VSIA was chosen because it produces opioidmediated analgesia with an abrupt onset and offset (2) and can be administered just prior to and during a tail-flick latency test, thereby eliminating any problems associated with the waning of morphine-induced analgesia over time.

\section{METHOD}

Forty-four female Long-Evans rats with normal estrous cycles were used. All rats were maintained in a colony with a $14 \mathrm{hr}$ on/10 $\mathrm{hr}$ off light/dark cycle with the onset of the light at $0500 \mathrm{hr}$ (EST). Rats were housed individually in $24.5 \times 18 \times 18$-cm wire-mesh cages. Food (Agway Prolab Rat/Mouse/Hamster Formula 3000) and water were available ad lib except during testing. Prior to testing, all rats were habituated to the test procedures: each was intubated without infusion and restrained for 1 hour/day for five consecutive days. Vaginal smears were obtained daily; tests were conducted only during diestrus.

Analgesia levels were measured using a tail-flick latency test. The "tail-flick" algesiometer used was similar to those used in other laboratories $(1,3,11)$; the details of our apparatus and procedures have been published previously (6). Tail-flick latency (TFL), measured in seconds, was the dependent variable. If no response occurred within $8 \mathrm{sec}$, the stimulus was terminated to avoid tissue damage. Rats were restrained in opaque polyvinyl

${ }^{1}$ Requests for reprints should be addressed to Dr. Mark B. Kristal, Department of Psychology, State University of New York at Buffalo, Buffalo, NY 14260 . 
chloride tubes $(5 \times 21 \mathrm{~cm})$ during the TFL test. Vaginal/cervical stimulation (VS) was produced by probing the vagina with a glass rod protruding from the barrel of a $1 \mathrm{cc}$ glass tuberculin syringe. The rod was spring loaded and calibrated to deliver $75 \mathrm{~g}$ of pressure (2).

Each measure consisted of two TFL tests. The first test score was the mean of the last 3 of 4 tail-flick trials without vaginal stimulation (TFL Baseline). This was immediately followed by the second test, in which the score consisted of the mean of 3 tail-flick trials with a constant vaginal stimulation (TFL + VS Baseline). Trials were separated by $30-\mathrm{sec}$ intervals. Percent change from TFL Baseline to TFL + VS Baseline was determined for each rat. The mean percent change from TFL Baseline was computed for each group to determine the extent of the analgesia produced by VS.

All subjects were food- and water-deprived for 3 hours prior to and during testing. Testing occurred during the light phase of the light/dark cycle, between 1000 and $1300 \mathrm{hr}$ (EST). Immediately after determining TFL + VS Baseline, half the subjects were intubated with $0.25 \mathrm{ml}$ amniotic fluid and half were intubated with $0.25 \mathrm{ml}$ beef bouillon. This dose of amniotic fluid was chosen because it was found to produce the greatest degree of enhancement of morphine-induced analgesia (6). Intubations were administered through a blunted 16-ga hypodermic needle fitted with a $11.5-\mathrm{cm}$ length of PE 160 tubing and mounted on a 1-cc glass syringe. Techniques for the collection and storage of amniotic fluid have been described elsewhere $(6,7)$. Beef bouillon (Wyler's Instant) was prepared according to package instructions at $1 / 2$ the recommended concentration and treated identically to amniotic fluid. For 26 rats, postintubation tests were conducted at 10, 20, $30,40,50$, and 60 minutes (repeated measures). After the completion of the above study, an additional eighteen rats, housed, maintained, and treated as described above, were given a postintubation test at 5 minutes.

\section{RESULTS}

Vaginal/cervical stimulation produced a mean increase in tail-flick latency of $8.23 \pm 2.74 \%$. Since the pressure used ( $75 \mathrm{~g})$ was near threshold (2), the increase in latency was small, but was statistically significant, $t(43)=3.00, p<0.05$. Approximately $33 \%$ of the rats tested for VSIA baseline (preintubation) were nonresponders (2). These were roughly evenly divided between the AF and $\mathrm{BB}$ groups. After intubation, all the nonresponders receiving $\mathrm{AF}$ became responders, whereas almost all the nonresponders receiving $\mathrm{BB}$ remained unresponsive to the VS.

The results from the first group are depicted in Fig. 1. A two-way ANOVA indicated a significant Fluid $\times$ Time interaction, $\mathrm{F}(4.58,109.97)=2.41, p<0.05$ (with a Greenhouse-Geisser adjustment), a significant main effect of fluid, $F(1,24)=6.71$, $p<0.05$, and a significant main effect of time, $\mathrm{F}(4.58,109.97)=$ $7.82, p<0.05$ (with a Greenhouse-Geisser adjustment). Further probing indicated a significant enhancement of VSIA by amniotic fluid ingestion occurring at 10,20 , and 30 minutes postintubation (Newman-Keuls, $p<0.05$ ), but not at 40,50 , or 60 minutes postintubation $(p>0.05)$. There were no significant differences in percent change from TFL + VS Baseline (i.e., no enhancement of VSIA) in rats intubated with beef bouillon (Newman-Keuls, $p>0.05$ ).

A $t$-test on the percent change from TFL + VS Baseline in the group receiving a 5-minute postingestion test revealed a significant enhancement at that time by amniotic fluid $(\mathrm{AF}=+28.46 \pm 8.1 \%$; $\mathrm{BB}=+2.84 \pm 9.07 \%), t(16)=2.11, p<0.05$. Therefore, POEF activity, measured as enhancement of VSIA after ingestion of amniotic fluid, is detectable by 5 minutes after ingestion, and lasts

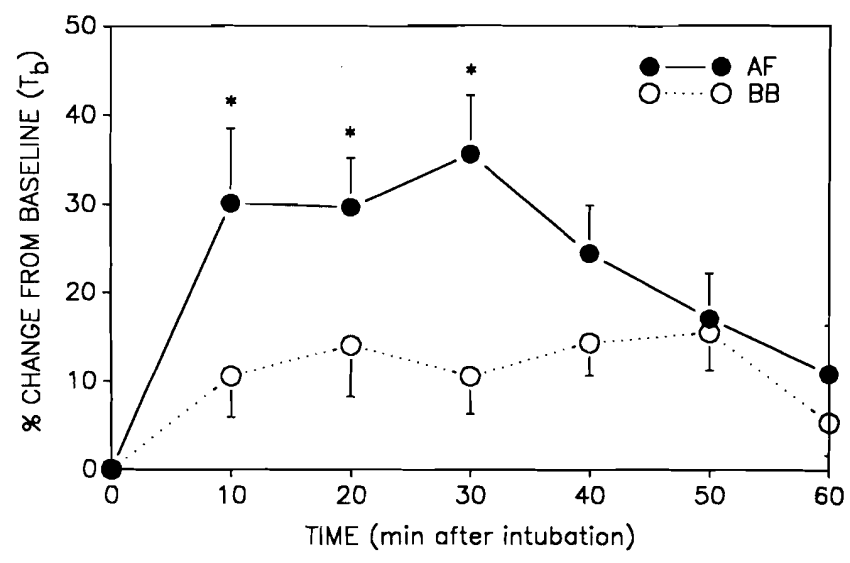

FIG. 1. Mean percent change from baseline vaginal/cervical stimulationinduced analgesia $\left(T_{b}\right)$ of rats that received either $0.25 \mathrm{ml}$ amniotic fluid (AF) or $0.25 \mathrm{ml}$ beef bouillon (BB) by gastric intubation before testing $\left(\mathrm{N}=26 ;{ }^{*} p<0.05\right)$.

between 30 and 40 minutes.

\section{DISCUSSION}

The above results demonstrate that amniotic fluid, in a dose similar to that contained in a term amniotic sac, enhances opioid-mediated analgesia produced by vaginal/cervical stimulation. These results are consistent with our previous reports on the enhancement of VSIA by the ingestion of placenta (10) as well as the enhancement of morphine-induced analgesia by ingestion of amniotic fluid $(6,7)$. We have further demonstrated that the enhancement begins within 5 minutes following ingestion, and lasts at least 30 minutes, but no longer than 40 minutes. The rapid onset of enhancement suggests either an extremely rapid absorption of POEF, or the possibility of activation of gastric neural receptors (6). Studies are underway to try to distinguish between these alternatives.

At the end of pregnancy there is an increase in endogenous opioids as well as an increase in opioid-mediated analgesia (pregnancy-mediated analgesia) (4). Amniotic fluid becomes available to the parturient female just prior to delivery; therefore, one of the benefits of ingesting amniotic fluid would be to enhance opioid-mediated analgesia at this time. Since the enhancement occurs within 5 minutes of ingestion and lasts at least 30 minutes, this would result in analgesia prior to delivery and prior to the availability of placenta. Subsequent ingestion of amniotic fluid or placenta after delivery, or between deliveries (which, in rats, occur at $20-30-\mathrm{min}$ intervals), would result in continued enhancement of opioid-mediated analgesia. The enhancement of VSIA by amniotic fluid has special relevance to the naturalistic situation since vaginal/cervical stimulation is a critical feature of parturition. Thus, amniotic fluid ingestion enhances opioid-mediated analgesia produced by vaginal/cervical stimulation, is available early during the birth process, is effective within 5 minutes, lasts between 30 and 40 minutes, and may be an important mediator of pain response during labor and delivery, and in the immediate postpartum period.

\section{ACKNOWLEDGEMENT} M.B.K.

This research was supported by NSF grant BNS86-01818 awarded to 


\section{REFERENCES}

1. Bolles, R. C.; Fanselow, M. S. Endorphins and behavior. Annu. Rev. Psychol. 33:87-101; 1982.

2. Crowley, W. R.; Jacobs, R.; Volpe, J.; Rodriguez-Sierra, J. F. Komisaruk, B. R. Analgesic effect of vaginal stimulation in rats: Modulation by graded stimulus intensity and hormones. Physiol. Behav. 16:483-488; 1976

3. D'Amour, F. E.; Smith, D. L. A method for determining loss of pain sensation. J. Pharmacol. Exp. Ther. 72:74-79; 1941

4. Gintzler, A. R. Endorphin-mediated increases in pain threshold during pregnancy. Science 210:193-195; 1980.

5. Kristal, M. B. Placentophagia: A biobehavioral enigma (or De gustibus non disputandum est). Neurosci. Biobehav. Rev. 4:141-150; 1980.

6. Kristal, M. B.; Abbott, P.; Thompson, A. C. Dose-dependent enhancement of morphine-induced analgesia by ingestion of amniotic fluid and placenta. Pharmacol. Biochem. Behav. 31:351-356; 1988.
7. Kristal, M. B.; Thompson, A. C.; Abbott, P. Ingestion of amniotic fluid enhances opiate analgesia in rats. Physiol. Behav. 38:809-815; 1986.

8. Kristal, M. B.; Thompson, A. C.; Abbott, P.; Di Pirro, J. M.; Ferguson, E. J.; Doerr, J. C. Modification of periparturitional pain threshold by ingestion of amniotic fluid in the rat. Soc. Neurosci. Abstr. 14(Part 2):1158; 1988.

9. Kristal, M. B.; Thompson, A. C.; Grishkat, H. L. Placenta ingestion enhances opiate analgesia in rats. Physiol. Behav. 35:481-486; 1985.

10. Kristal, M. B.; Thompson, A. C.; Heller, S. B.; Komisaruk, B. R. Placenta ingestion enhances analgesia produced by vaginal/cervical stimulation in rats. Physiol. Behav. 36:1017-1020; 1986.

11. Taber, R. I. Predictive value of analgesic assays in mice and rats. In: Braude, M. C.; Harris, L. S.; May, E. L.; Smith, J. P.; Villarreal, J. E., eds. Advances in biochemical pharmacology, vol. 8: Narcotic antagonists. New York: Raven Press; 1974. 